\title{
Investigation of the influence of wall stiffness on the stress ratio in mammoth silos
}

\author{
P.L.L. van Leeuwenstijn ${ }^{a}$, L.A. van Wijk ${ }^{b}$ and G. Haaker ${ }^{a}$ \\ "Faculty of Mechanical Engineering, University of Twente, PO Box 217, 7500 AE Enschede (The Netherlands) \\ ${ }^{b}$ ESI-Engineers and Contractors BV, PO Box 1047, 1440 BA Purmerend (The Netherlands)
}

(Received April 27, 1993; accepted October 25, 1993)

\begin{abstract}
To calculate the stresses on the walls of silos, it is necessary to have a good estimate of the ratio of horizontal to vertical stress. This ratio however is not known precisely, especially in cases of static stress as found in a mammoth silo. In this paper the influence of the wall stiffness on the ratio of horizontal to vertical stresses in situations with limit strain is investigated. A new test method is developed, with which the ratio can directly be measured. A key feature of this method is the comparability with the practice. The method uses reinforced rubber membranes which have the same order of radial stiffness as mammoth silos. Another objective was to determine the extent to which the ratio of horizontal to vertical stresses with limit strain is dependent on the internal angle of friction, $\phi_{\mathrm{i}}$. Special attention was paid to Jaky's formula, which is often used to calculate the neutral stress ratio.
\end{abstract}

\section{Introduction}

Theory concerning stresses in slender silos commonly distinguishes two extremes: an active and a passive stress state, in which the major principal stress, $\sigma_{1}$, is vertically or horizontally orientated, respectively. In both cases the bulk is assumed to be in limit yield state.

The stresses occurring on filling a slender silo are not so important with respect to design. The method of discharging these silos makes the stresses at discharge much higher than those at filling and therefore more relevant for design [1]. In mammoth silos, however, the method of discharging involves scraping off the surface of the bulk, a low-stress operation. Therefore, in contrast, stresses during filling are higher. This is recognized in the German standard DIN 1055, which states that with a height-to-diameter ratio smaller than 0.8 , wall pressures must be calculated according to retaining wall theory, at rest [8]. The values of these stresses then become interesting for economic reasons.

It is known from retaining-wall theory that a bulk solid will only be in an active state when there is sufficient horizontal strain. The absence of horizontal strain is defined as neutral in geotechnics, the corresponding stress ratio being $K_{\mathbf{0}}$. In geotechnics this ratio is often predicted using Jaky's formula $[2,4]$ :

$K_{0}=1-\sin \left(\phi_{\mathrm{i}}\right)$
Another prediction has been made by Miller [2, 5]. However, for certain values of $\phi_{\mathrm{i}}, K_{0}<K_{\mathrm{a}}$ [7] which is physically impossible.

Strains in a bulk solid are dependent on the stiffness of the wall. Due to the finite stiffness, the strain in the wall is small. Thus the strains are not large enough to assume an active stress state, and yet they may not be small enough to presume a neutral state. Nevertheless all simple thcories arc based on the assumption of an active state.

Recently Wijk [9] showed that application of Coulomb's theory beyond its theoretical foundation, by taking the neutral angle of friction instead of the active one, is not satisfactory. He showed how the assumption of the backfill of a retaining wall, being in the limit yield state, can be bypassed while still keeping the solution simple and easy to manage. The model relates five ratios in stresses, describing the stress state by two equations obtained from equilibrium. To determine the stress state fully, three additional assumptions are necessary. Two of these missing equations, which are in this case the two ratios being determined, may still originate from the assumption of the backfill being active near the wall.

The results are close to those predicted by the Coulomb theory if for the fifth parameter, which is the stress ratio far from the wall, the active stress ratio $K_{\mathrm{a}}$ is taken. The advantage of the new model, however, is that the latter assumption can be dropped by assigning 
the fifth parameter a value more representative for the undisturbed material.

This paper investigates how the ratio of horizontal to vertical stresses with limited horizontal strain, is dependent on the wall stiffness as found in practice. This ratio is denoted by $K_{\mathrm{s}}$. Another issue in this survey is the correlation of $\mathrm{K}_{0}$ with the internal angle of friction, $\phi_{\mathrm{i}}$. The existence of a smooth relation between $K_{\mathrm{s}}$, as substitute for $K_{0}$, and $\phi_{\mathrm{i}}$ is examined.

\section{Experimental}

\section{Design of apparatus}

At the Bulk Solid Laboratory of the University of Twente a new triaxial tester has been developed [7]. The method uses a tangentially reinforced, free-standing rubber membrane. Axially the membrane is flexible, which prevents development of shear stresses at the membrane-bulk interface. Radially the membrane is stiff because of the presence of the reinforcement, which consists of isotan electrical resistance wire, wound in the membrane with a small pitch (Fig. 1).

The membrane encases a sample of a bulk solid which is axially loaded. The strain in the wire, $\epsilon_{\mathrm{w}}$, is measured by the resistance increase $(\Delta R)$. It can be seen that the strain in the circumferential direction equals that in the radial direction:

$\epsilon_{\mathrm{w}}=\epsilon_{\mathrm{r}}$

The stress in the wire, $\sigma_{\mathrm{w}}$, is related to the radial stress, $\sigma_{\mathrm{r}}$, on the membrane by the equilibrium (Fig. 2):

$\sigma_{\mathrm{r}}=\frac{\frac{\pi}{4} \cdot d^{2}}{\frac{1}{2} \cdot D \cdot s} \cdot \sigma_{\mathrm{w}}$

in which $d$ is the diameter of the wire, $D$ the diameter of the membrane and $s$ the pitch.

The relation between the stress and the strain in the wire is:

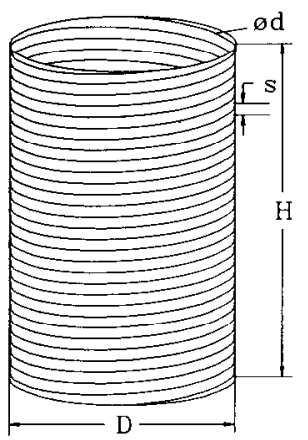

Fig. 1. Reinforced rubber membrane.
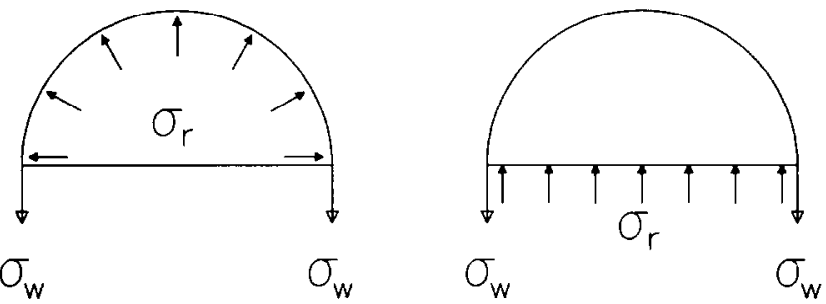

Fig. 2. Force equilibrium exerted on the membrane.

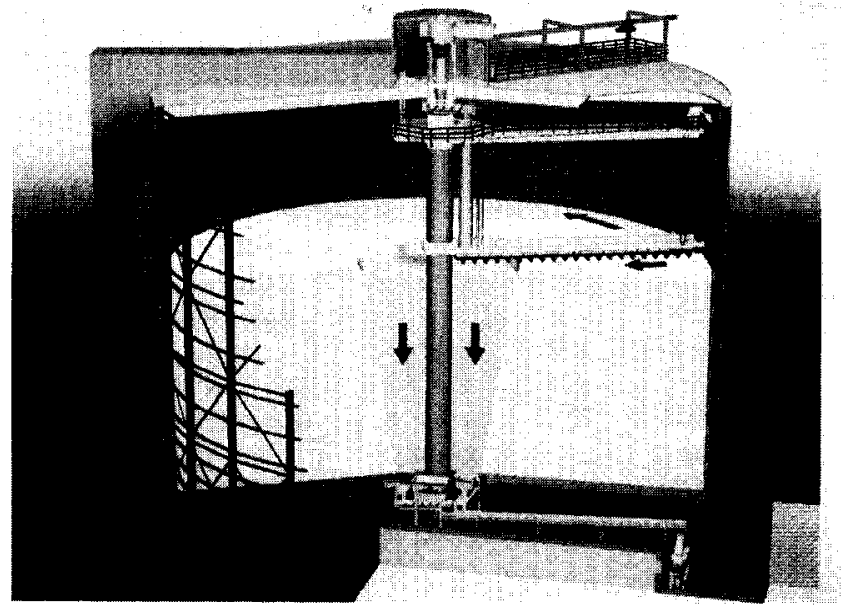

Fig. 3. Mammoth silo of the Eurosilo type.

$\sigma_{\mathrm{w}}=E_{\mathrm{w}} \cdot \epsilon_{\mathrm{w}}$

with $E_{w}$ being the Young's modulus of the wire.

The radial stiffness is defined as:

$E_{\mathrm{r}}=\frac{d \sigma_{\mathrm{r}}}{d \epsilon_{\mathrm{r}}}$

With $\bar{\sigma}_{\mathrm{r}}$ as the design value of the radial stress and $\bar{\sigma}_{w}$ as the design value of the wire stress, the radial stiffness can be expressed as:

$E_{\mathrm{r}}=E_{\mathrm{w}} \frac{\bar{\sigma}_{\mathrm{r}}}{\bar{\sigma}_{\mathrm{w}}}$

In mammoth silos of the Eurosilo type, the walls are supported by horizontal ring-beams which assume the horizontal load (Fig. 3). For these silos the design radial stress and the design stress in the ring beam are related by a formula analogous to eqn. (3) resulting in eqn. (6). The order of magnitude of radial stiffness of membranes and silos is the same. A representative magnitude of $E_{\mathrm{r}}$ is 65 to $225 \mathrm{~N} \mathrm{~mm}^{-2}$.

In order to obtain a specific stiffness, the design parameters of the membrane, which are the reinforcing wire diameter, $d$, and the pitch, $s$, can be varied. For the diameter, $d$, two values were used: $0.15 \mathrm{~mm}$ and $0.20 \mathrm{~mm}$. A smaller diameter, $0.11 \mathrm{~mm}$, results in a fragile membrane, which is easily deformed, and in which the stress level in the wires is too high. A large 
diameter results in the opposite; the stress level is too low to obtain good resolution in strain measurements. The pitch was varied from $0.5 \mathrm{~mm}$ to $1 \mathrm{~mm}$. A pitch smaller than $0.5 \mathrm{~mm}$ is problematic since the wires can touch each other; larger than $1 \mathrm{~mm}$ may influence the measurement of $K_{\mathrm{s}}$. The influence of pitch is discussed later.

The diameter of the membrane, $D$, was $100 \mathrm{~mm}$ and the height about $230 \mathrm{~mm}$. Neither value was varied. These values provided the best combination of accuracy and utility.

The stress in the wires, $\sigma_{w}$, causes an increase in electrical resistance of the wire. The resistance increase, $\Delta R$, of the initial value of $R(=120 \Omega)$, was measured with a Wheatstone bridge. The relation between the bridge disturbance $\Delta V / V$ and the circumferential stress, $\sigma_{\mathrm{w}}$, is [6]:

$\frac{\Delta V}{V}=\frac{C}{4} \cdot \epsilon_{\mathrm{w}}$

With eqn. (4) this can be written as:

$\frac{\Delta V}{V}=C^{\prime} \cdot \sigma_{\mathrm{w}} \quad$ with $C^{\prime}=\frac{C}{4 E_{\mathrm{w}}}$

where $C^{\prime}=$ material constant, in $\mathrm{Pa}^{-1}$.

The part of the circumferential force carried by the latex is negligibly small. The circumferential strain is small $(\mathrm{O}(-4))$ and the Young's modulus of latex compared to isotan is small (latex: $1.6 \times 10^{6} \mathrm{~Pa}$, isotan: $1.7 \times 10^{11} \mathrm{~Pa}$ ). The cross-sectional areas of latex and wire differ by one order of magnitude, so the force is virtually completely carried by the wire.

Equation (3) gives the relation between the radial stress $\left(\sigma_{\mathrm{r}}\right)$ and the circumferential stress $\left(\sigma_{\mathrm{w}}\right)$. This relation is based on stretched dimensions. The radial and circumferential strains are small compared to the axial strain $\left(\epsilon_{\mathrm{r}}=\epsilon_{\mathrm{w}} \leqslant 10^{-4}, \epsilon_{\mathrm{a}} \leqslant 10^{-2}\right)$, so they can be neglected. Based on unstretched dimensions, and expressing $\sigma_{\mathrm{w}}$ as a function of $\sigma_{\mathrm{r}}$, the relation becomes:

$\sigma_{\mathrm{w}}=\sigma_{\mathrm{r}} \cdot \frac{2 \cdot D \cdot s\left(1+\epsilon_{\mathrm{a}}\right)}{\pi \cdot d^{2}}$

Substituting eqn. (9) into eqn. (8), the relation between the bridge disturbance and the radial stress is obtained as:

$$
\begin{aligned}
\frac{\Delta V}{V} & =\sigma_{\mathrm{r}} \cdot \frac{C \cdot D \cdot s\left(1+\epsilon_{\mathrm{a}}\right)}{E_{\mathrm{w}} \cdot 2 \cdot \pi \cdot d^{2}} \\
& =C^{\prime \prime} \cdot\left(1+\epsilon_{\mathrm{a}}\right) \cdot \sigma_{\mathrm{r}}, \text { with } C^{\prime \prime}=\frac{C \cdot D \cdot s}{E_{\mathrm{w}} \cdot 2 \cdot \pi \cdot d^{2}}
\end{aligned}
$$

This relation is used to calculate $\sigma_{\mathrm{r}}$ from the measured bridge disturbance. Later it will be shown how the constant $C^{\prime}$, and thus $C^{\prime \prime}$ (eqn. (10)), was estimated.

\section{Manufacture of membrane}

The membranes were fabricated on a cylindrical mould. This generated two problems. The major problem was the reinforcement, which is applied to the membrane on the mould with a certain prestress. Because of this stress a compressive force was introduced. All the contributing windings resulted in a compressive force of the order of $500 \mathrm{~N}$.

The second problem was the stickiness of the latex, which, together with the compressive force, makes it impossible to remove the membrane from the mould without damaging it. The first step in solving this twophase problem is to use a mould with a reducible diameter. The mould is built up in five parts; two top, two side and a central part (Fig. 4). After removing the central part, the compressive force drives the two side parts inward. This way the compressive force is eliminated. A thin plastic sheet is placed between the mould and the membrane. After production, the membrane, on the sheet, can easily be removed from the mould. The sheet, which sticks to the latex, can now carefully be detached from the membrane. The quality of the membranes produced this way is good. The mould with plastic sheet is placed on a lathe. This has several advantages: first, using the thread-cutting attachment of the lathe makes it possible to establish a constant pitch and, secondly, it makes it easy to apply the liquid latex solution on the mould. The membrane production process consists of three steps: first the ground layer of latex is applied. Secondly, the wire is wound and finally the outer layer of latex is applied. After producing the membrane, the mould is taken apart and the sheet is removed from the membrane.

Dried latex remains slightly sticky. To neutralize this, talcum powder is sprinkled on both sides of the membrane. After removing the membrane from the mould, the pitch shrinks slightly (ca. 6\%) by contraction of the latex due to drying. However, this does not affect the usefulness of the membranes.

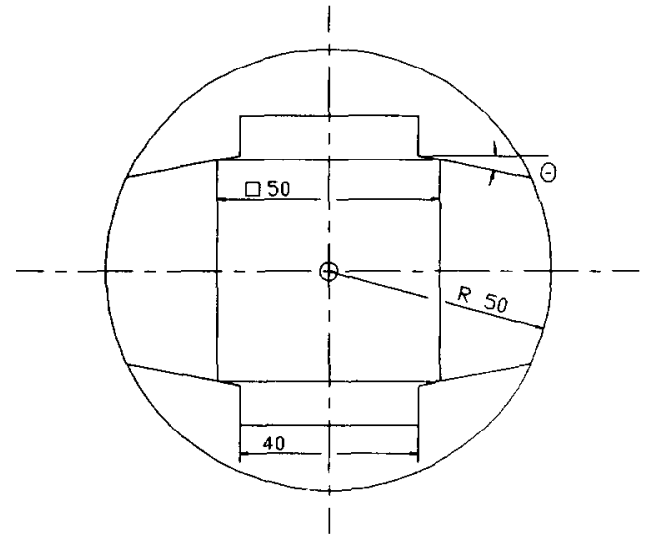

Fig. 4. Cylindrical mould. 
The actual value of the pitch is denoted as $s_{\mathrm{m}}$. The values of radial stiffness obtained for the membranes by using different combinations of wire diameter, $d$, and pitch, $s$ and $s_{\mathrm{m}}$, are given in Table 1 .

A last remark should be made concerning the preservation of the membranes. The latex is sensitive to UV-radiation which ages the membranes; the latex loses its flexibility and becomes brittle. To avoid this, the membranes should be stored in the dark.

\section{Calibration of apparatus}

The relation between the bridge disturbance and the radial stress consists of two parts (eqn. (10)), a membrane-geometry constant, containing $\mathrm{d}$ and $\mathrm{s}$ replaced by $s_{\mathrm{m}}$ and $D$, and a material constant, $C^{\prime}$.

$C^{\prime}$ could be calculated (eqn. (8)), but it is better to measure it directly since the accuracy of the Young's modulus given for isotan is not known. The membranedependent part can be calculated. Thus the relation between the bridge disturbance and the radial stress can be calculated using the first part of eqn. (10).

Another method to establish the relation between the bridge disturbance and the radial stress is to measure $C^{\prime \prime}$ directly, using the second part of eqn. (10). The first method however is preferable, since $C^{\prime}$ is materialdependent only and the membrane-dependent part can be calculated. To check the validity of the first method, the second method is used.

The first method uses eqn. (8). $C^{\prime}$ can be estimated by applying a load to the wire and measuring the bridge disturbance. The wire is suspended in a crane and, in unloaded condition, $120 \Omega$ is integrated in the Wheatstone bridge. Then the wire is loaded in several load steps by hanging weights (ca. $50 \mathrm{~g}$ ) on the wire. The load is measured with a load cell. After each step the bridge disturbance and the load are measured. The value of $C^{\prime}$ estimated this way is:

$C^{\prime}=3.80 \times 10^{-12} \frac{V / V}{\mathrm{~Pa}} \pm 1.9 \%$

With the second method the membrane is directly loaded with a bellows inflated by air pressure. The air pressure is measured using a mercury column. The relation is verified in two ways: with suppressed axial

TABLE 1. Design pitch $s$ and real pitch $s_{m}$ values with the resulting radial stiffness

\begin{tabular}{lllll}
\hline$s$ & $d$ & $s_{\mathrm{m}}$ & $d$ & $\begin{array}{l}E_{\mathrm{r}} \\
\left(\mathrm{N} \mathrm{mm} \mathrm{mm}^{-2}\right)\end{array}$ \\
\hline 0.20 & 0.50 & 0.20 & 0.47 & 225.8 \\
0.15 & 0.50 & 0.15 & 0.47 & 127.1 \\
0.15 & 0.75 & 0.15 & 0.70 & 85.0 \\
0.15 & 1.0 & 0.15 & 0.94 & 64.3 \\
\hline
\end{tabular}

strain, $\epsilon_{\mathrm{a}}=0$, and with axial strain, $\epsilon_{\mathrm{a}} \neq 0$. With suppressed axial strain $C^{n}$ can directly be measured, and with axial strain the correction for strain (eqn. (10)) can be verified.

It is not possible to suppress axial strain by fixing the ends of the bellows because this will cause the bellows to buckle. Instead of fixing the ends, a sheet, axially cut in strips 5 to $10 \mathrm{~mm}$ wide, is placed between the bellows and the membrane. To check whether the sheet has any influence, tests were done with sheet cut in 5 or $10 \mathrm{~mm}$ strips and with continuous sheet (no strips). The results obtained with 5 and $10 \mathrm{~mm}$ strips were identical, both in a range of $\pm 0.5 \%$ of one another. The test with continuous sheet resulted in a maximum output level that was $10 \%$ lower than the tests with sheet cut in strips. So when the sheet is cut in small strips, 5 to $10 \mathrm{~mm}$ wide, the sheet does not influence the measurements. Comparing method two $\left(\epsilon_{\mathrm{a}}=0\right)$ with method one gives good agreement. The difference between the measured value of $C^{\prime \prime}$ (method two) and the calculated value of $C^{\prime \prime}$ (method one, eqn. (10)) is less than $1 \%$. So with suppressed strain, calculating $C^{\prime \prime}$ on the basis of $C^{\prime}$ is allowed.

When axial strain is not suppressed, it attains a valuc up to $8 \%$. The calculated value of $C^{\prime \prime}$ is now ca. $10 \%$ smaller than with suppressed strain. The explanation of this phenomenon lies in the fact that the bellows is made of latex, reinforced with nylon. When straining axially, a radial stress component is introduced by Poisson's ratio $\nu$ [3]. This component carries a part of the radial load, which results in a lower value of $C^{\prime \prime}$ [7]. Thus when calibrating the membrane directly, it is advisable to suppress axial strain.

In actual, tests the influence of the axial strain is small, because the strain is negative (the sample is compacted) and any build-up stress is only introduced in the membranc shell, which is not axially reinforced and is thin $(<0.2 \mathrm{~mm})$, so the force will be small.

\section{Test procedure}

Figure 5 illustrates the experimental set-up. The membrane is tightly wrapped around a load cell, which measures the axial stress, without resting on the cell itself. The membrane is now filled to $1 \mathrm{~cm}$ from the top. The filling procedure is dependent on the character of the material.

For free-flowing material the procedure is simple: the material is poured into the membrane. Cohesive materials however must be prepared carefully, especially when using aeratable materials. The mombrane is fillcd in layers of $2-3 \mathrm{~cm}$. Each layer is preconsolidated with $3 \mathrm{kPa}$ to deaerate the material.

After filling the membrane, the top platen is placed on the bulk. In the middle of the platen a steel ball is placed, on which the actual load rests. This steel 


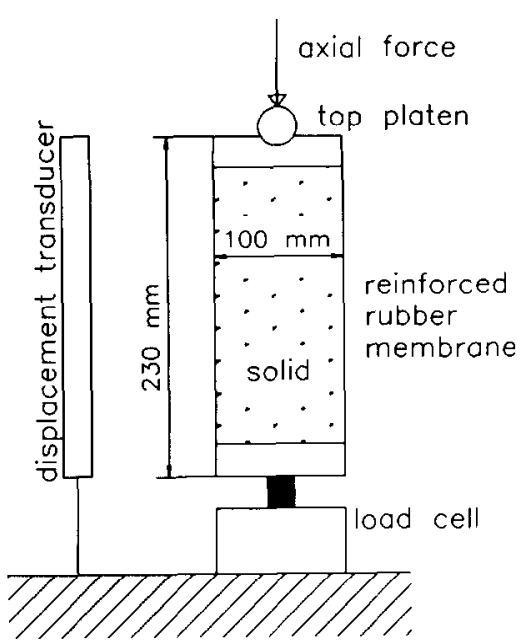

Fig. 5. Experimental set-up of the triaxial test.

ball prevents the development of a momentum in the sample. Both the membrane and the load cell are connected to a programmable data recorder through a Wheatstone bridge. The required voltage is supplied by an interface which has a fecding voltage of $5 \mathrm{~V}$ DC.

The strain is measured by means of an external displacement transducer, which is connected to the data recorder through a power amplifier with a voltage of $5 \mathrm{~V} \mathrm{AC}$. The data recorder is attached to a personal computer. Using this configuration makes it possible to take the data, transfer it directly to the PC and process it immediately. Thus the data can be corrected for axial strain instantly. The computer can control the data-gathering process and is programmed to take a sample every $25 \mathrm{~s}$, or about every $2 \mathrm{kPa}$ in vertical stress.

The load ratio $K_{\mathrm{s}}$ can be calculated by drawing a graph of the measured stresses, $\sigma_{\mathrm{a}}$ and $\sigma_{\mathrm{r}} . K_{\mathrm{s}}$ is the tangent of the line. The accuracy of the system, containing the data recorder, the computer, the power amplifier and the DC power unit, is better than $97.3 \%$.

The load is stress-driven and consists of water which is pumped into a barrel resting on the sample. The load is applied via a lever system. After balancing the lever, it touches the sample but does not lean on it. Then the offset values of the channels, used to measure $\sigma_{\mathrm{a}}, \sigma_{\mathrm{r}}$ and $\epsilon_{\mathrm{a}}$, are recorded and water is poured into the barrel. Each $25 \mathrm{~s}$ a sample is taken on each channel. This continues until the maximum load of about 50 $\mathrm{kg}$, which equals $65 \mathrm{kPa}$, is reached. Now the process is reversed, water is poured out of the barrel. In both loading and unloading, sample readings are taken.

\section{Test program}

Before the actual investigation was performed, the influence of the pitch was examined. Two membranes
TABLE 2. Min/Max deviation of cach test from the mean valuc of the 4 measured values of $K_{\mathrm{s}}$

\begin{tabular}{llllll}
\hline Material & $K_{\mathrm{s}, \text { mean }}$ & I & I & II & II \\
\hline Silversand & 0.4475 & $-0.1 \%$ & $+1.4 \%$ & $+0.6 \%$ & $-1.9 \%$ \\
Detergent & 0.328 & $-0.9 \%$ & $0.0 \%$ & $+0.9 \%$ & $0.0 \%$ \\
\hline
\end{tabular}

with corresponding stiffness but different pitch were used to measure $K_{\mathrm{s}}$ for silversand and detergent. Each test was performed twice.

$$
\begin{aligned}
& \text { Membrane I: } 0.15 \times 0.47, E_{\mathrm{r}}=127.0 \mathrm{~N} \mathrm{~mm}^{-2} \text {; } \\
& \text { Membrane II: } 0.20 \times 0.94, E_{\mathrm{r}}=114.2 \mathrm{~N} \mathrm{~mm}^{-2} \text {. }
\end{aligned}
$$

Table 2 shows clearly that the measured values of $K_{\mathrm{s}}$ vary little. It is thus concluded that for actual values of $s_{\mathrm{m}}(0.5-1 \mathrm{~mm})$ the pitch has no influence on $K_{\mathrm{s}}$.

The test program consists of two parts, one to examine the influence of the wall stiffness and the other to investigate the correlation of $K_{\mathrm{s}}$ with $\phi_{\mathrm{i}}$. $K_{\mathrm{s}}$ for seven materials was determined using four different membranes. The stiffnesses, $E_{\mathrm{r}}$, of the membranes were respectively: $64.3,85.0,127.0$ and $225.8 \mathrm{~N} \mathrm{~mm}^{-2}$. The tested bulk solids were silversand, salt, detergent, sodium hexacyanoferrate, synthetic grains, phosphate rock, and fertilizer. Although most of the bulk solids used are free-flowing, their natures differ. Silversand particles are smaller than $0.1 \mathrm{~mm}$ in diameter, while fertilizer particles can be up to $6 \mathrm{~mm}$ or more. Also the shape is different. Synthetic grains are almost spherical, while salt is more cubical.

To obtain further data concerning $K_{\mathrm{s}}$ and $\phi_{\mathrm{i}}$, three additional materials were tested with only one membrane: potato starch, plaster and lactose. These materials are cohesive and aeratable. Testing them takes much time, which is the reason that only one membrane was used to measure $K_{\mathrm{s}}$. The membrane used had a radial stiffness of $(0.15 \times 0.71), E_{\mathrm{r}}=85.0 \mathrm{~N} \mathrm{~mm}^{-2}$. Each test was performed twice. If the deviation was less than $2.7 \%$, the result was accepted, otherwise a third test was performed to determine more precisely the value of $K_{\mathrm{s}}$.

For all the solids used the internal angle of friction was measured with a standard Jenike shear cell. For comparison with $\phi_{\mathrm{i}}$, the value of $K_{\mathrm{s}}$ measured with the stiffest membrane was used to best approach $K_{0}$. Thus a relation bctween $K_{\mathrm{s}}$ and $\phi_{\mathrm{i}}$, if present, represents a relation between $K_{0}$ and $\phi_{i}$.

\section{Results}

A typical graph of the triaxial test is shown in Fig. 6. While loading the sample, the relation between $\sigma_{\mathrm{r}}$ and $\sigma_{\mathrm{a}}$ is linear. Upon unloading, however the material 


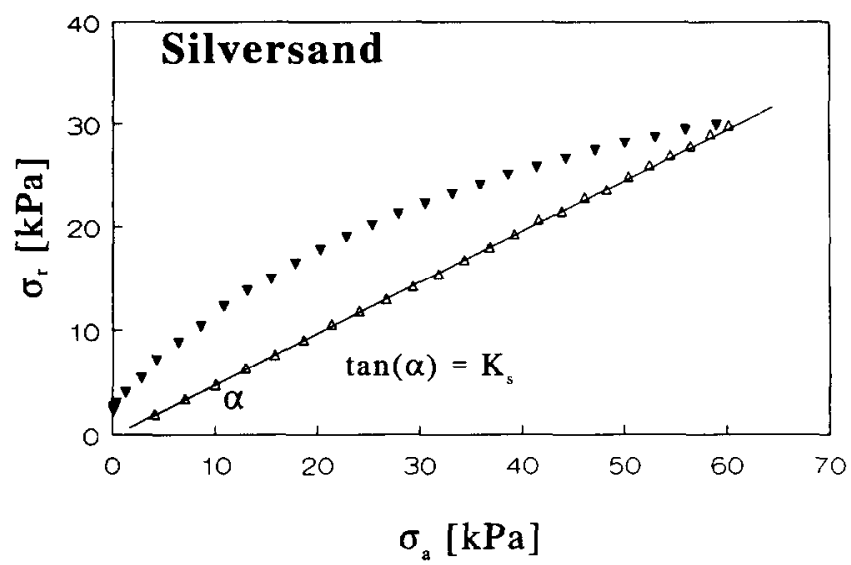

Fig. 6. Example of the loading-unloading curve, executed with a membrane with stiffness $E_{\mathrm{r}}=225.8 \mathrm{~N} \mathrm{~mm}^{-2}$. ( $\triangle$ ) Loading; $(\boldsymbol{\nabla})$ unloading.

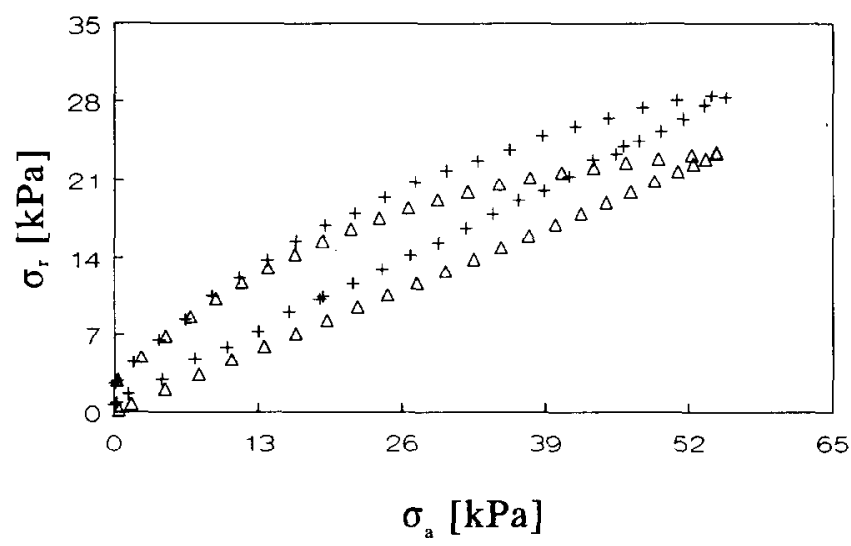

Fig. 7. Stress response measured with two membrane stiffnesses, $(+) E_{\mathrm{r}}=225.8 \mathrm{~N} \mathrm{~mm}^{-2}(0.20 \times 0.47)$ and $(\triangle) E_{\mathrm{r}}=64.3 \mathrm{~N} \mathrm{~mm}^{-2}$ $(0.15 \times 0.94)$.

shows considerable hysteresis. The radial stress, $\sigma_{\mathrm{r}}$, first diminishes slowly, but gradually starts to drop faster. When the axial load is less than $10 \%$ of the maximum load, $\sigma_{\mathrm{r}}$ starts to decrease rapidly. The slope of the unloading line represents $K_{\mathrm{p}}$.

During compaction the solid particles slide along each other and the density will increase. Before the radial stress can reverse this process upon unloading, the axial stress has to decrease substantially. Deformations are only partially recovered, much of them being irreversible.

A correlation of the hysteresis loop with cohesion is not observed, yet it seems that the loop is smaller with a stiffer membrane. This effect should be further investigated (Fig. 7).

The axial strain versus axial stress is given in Fig. 8. The compaction of the material is large while loading (up to 6\%) and nonlinear with the axial stress. A great part $(50-60 \%)$ of the maximum strain is reached during the first $13 \mathrm{kPa}$. The slope of the curve at just loading $(<13 \mathrm{kPa})$ diminishes to a reasonable constant value

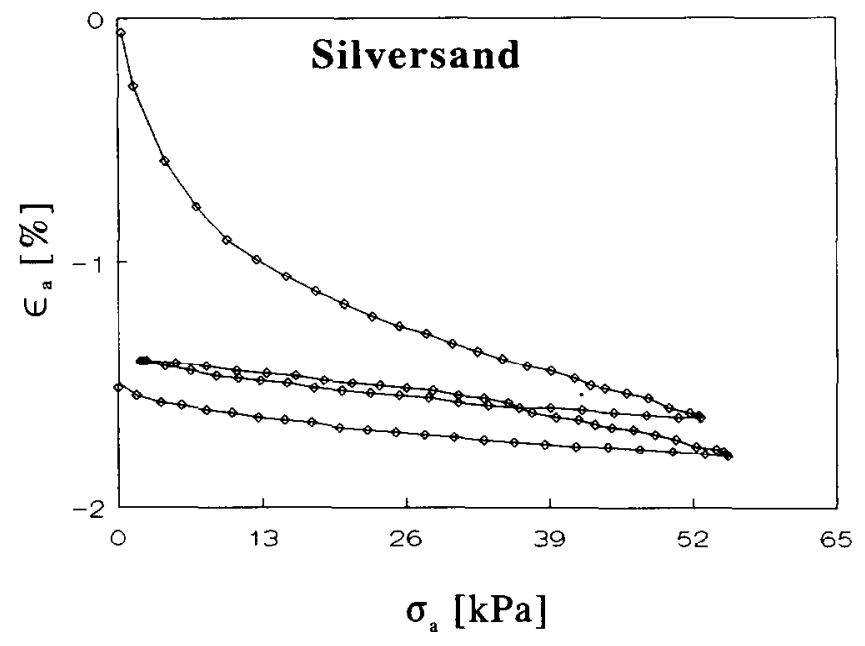

Fig. 8. Axial strain response of a sample.

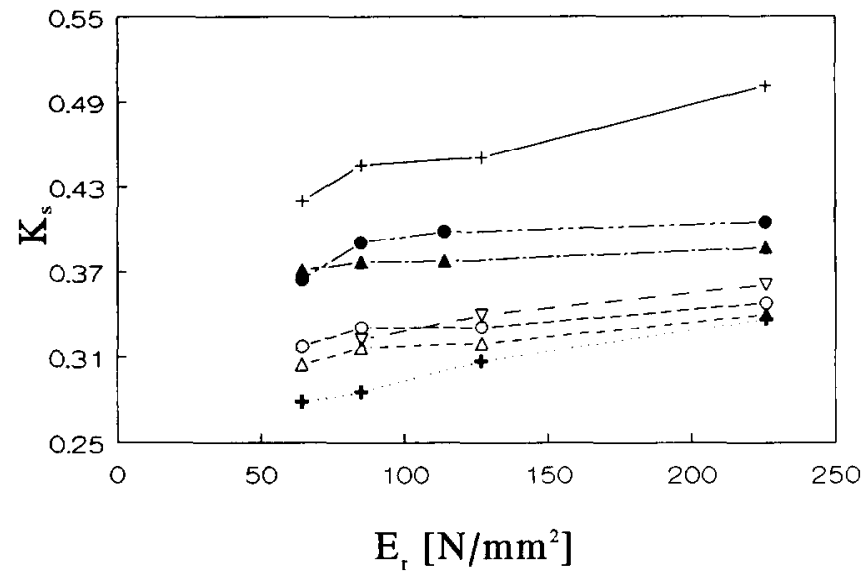

Fig. 9. Relation of $K_{\mathrm{s}}$ with the wall stiffness, $E_{\mathrm{r}}$, for seven materials: $(+)$ silversand, $(\triangle)$ salt, $(\Delta)$ synth. grains, $(O)$ detergent, $(\bullet)$ phosphate, $(+)$ sodium hexacyanoferrate, $(\nabla)$ fertilizer.

at large loads (13-65 kPa). This means that during the first $13 \mathrm{kPa}$ the stiffness of the material increases.

The deformations during initial loading are mainly plastic, since only a small part of the deformations are recovered while unloading, ca. $10 \%$ of the total. The tangent of the unloading curve is nearly constant. When the sample is reloaded, the tangent of the stress-strain curve corresponds fairly well with the tangent of the initial unloading curve, besides some hysteresis effects. The slope of the reloaded unloading line has approximately the same value as the slope of the initial unloading line (Fig. 8). The test program gives 30 pairs of measured $K_{\mathrm{s}}$ values. With this data the standard deviation of this test method can be calculated and is $1.7 \%$.

\section{Influence of the wall stiffness on $K_{s}$}

For all the materials tested, the relation of $K_{s}$ with the wall stiffness, $E_{\mathrm{r}}$, is presented in Fig. 9. It was 
found that with a weaker membrane the stress ratio decreases. This behavior was observed with all materials, however not to the same magnitude. For sand, $K_{s}$, measured with the stiffest membrane, is 0.500 and with the weakest membrane 0.420 , which means a decline of $16 \%$. Sodium hexacyanoferrate decreased the least: $7 \%$.

A possible explanation for this behavior is that with a weaker membrane, the material has more space to deform and initiate yielding. The stress ratio will therefore decrease and move in the direction of $K_{\mathrm{a}}$. This conduct can explain why the hysteresis loop of the $\sigma_{\mathrm{r}}-\sigma_{\mathrm{a}}$ graph seems to be smaller for stiffer membranes (Fig. 7). The radial stress for a stiffer membrane is higher than for a weaker one. Therefore the axial stress needs to diminish less before the radial stress is able to reverse the deformation process. The loop is therefore smaller.

\section{Relation of $K_{s}$ with the internal angle of friction}

The possible correlation of $K_{\mathrm{s}}$ with $\phi_{\mathrm{i}}$ was investigated using the values of $K_{\mathrm{s}}$ measured with the stiffest membrane $\left(E_{\mathrm{r}}=225.8 \mathrm{~N} \mathrm{~mm}^{-2}\right)$, because they are closest to $K_{0}$ (no radial strain). The gain of $K_{\mathrm{s}}$ with the internal angle of friction, $\phi_{i}$, is given in Fig. 10, together with Jaky's approximation. Because the last three solids were tested with a weaker membrane, a $10 \%$ increase is assumed as an upper limit. It shows clcarly that no smooth correlation between $K_{\mathrm{s}}$ and $\phi_{\mathrm{i}}$ is present. It is questionable whether a smooth relation between $K_{0}$ and $\phi_{i}$ exists. To compare Jaky's formula with a simple approximation of $K_{0}=0.4$ (as suggested by Jenike), both can be written as:

$K_{0}^{\text {measured }}=\left(1+\zeta_{0.4}\right) \cdot 0.4$

$K_{0}^{\text {measured }}=\left(1+\zeta_{\text {Jaky }}\right) \cdot\left(1-\sin \left(\phi_{\mathrm{i}}\right)\right)$

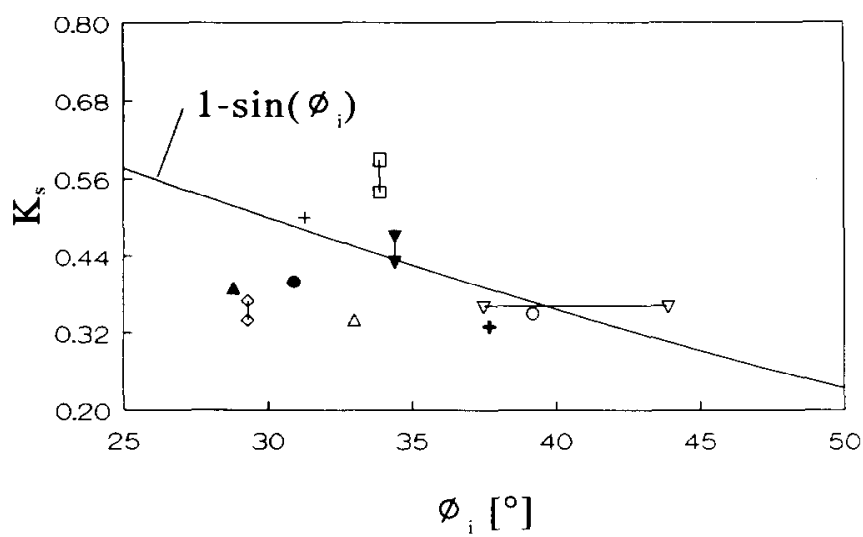

Fig. 10. Relation of $K_{\mathrm{s}}$ with the internal angle of friction, $\phi_{\mathrm{i}}$ for $(+)$ silversand, $(\odot)$ phosphate, $(\triangle)$ salt, $(\nabla)$ fertilizer, $(O)$ detergent, $(\square)$ potato starch, $(+)$ sodium hexacyanoferrate, $(\diamond)$ plaster, (A) synth. grains, ( $\boldsymbol{\nabla})$ lactose.
TABLE 3. The mean value and the standard deviation of correction factor $\zeta$ for $K_{0}=0.4$ and for Jaky's formula, based on $\phi_{i}$ and $\phi_{c}$

\begin{tabular}{lccc} 
& $K_{0}=0.4$ & $K_{0}=1-\sin \phi_{i}$ & $K_{0}=1-\sin \phi_{\mathrm{e}}$ \\
\hline Mean, \% & -0.5 & -7.7 & 8.5 \\
s.d., \% & 18.0 & 17.9 & 20.9
\end{tabular}

From our results the correction factor $\zeta_{0.4}$ can be calculated as having a mean value of $-0.5 \%$ and a standard deviation of $18.0 \%$. Based on the internal angle of friction $\zeta_{\mathrm{Jaky}, \phi i}$ has a mean value of $-7.7 \%$ and a standard deviation of $17.9 \%$. This means that Jaky has to be corrected by a factor 0.92 . Only then is the merit of the prediction made by this corrected Jaky formula equal to that of $K_{\mathrm{s}}=0.4$. Based on the effective angle of friction, $\zeta_{\text {Jaky, } \phi e}$ can be estimated as having a mean value of $8.5 \%$ and a standard deviation of $20.9 \%$. It is the standard deviation of $\zeta_{\text {Jaky, de }}$ that makes this approximation slightly less reliable than the approximation of $K_{0}=0.4$. Table 3 gives an overview of the figures. Thus, the use of Jaky's equation to predict the horizontal to vertical stress ratio in silo design is no better than the approximation $K_{0}=0.4$.

\section{Conclusions}

The test method as described has good repeatability, with a standard deviation of $1.7 \%$, even in the absence of a strict preparation procedure. This is remarkably low compared with the scatter that is generally experienced when measuring yield properties of bulk solids.

The obtained values for wall stiffness of the membranes agrees with the practical values of wall stiffness of mammoth silos. For these values, $K_{\mathrm{s}}$ is dependent on wall stiffness. A decrease of up to $16 \%$ in the $K_{\mathrm{s}}$ values at low wall stiffness was measured.

No correlation was found between the measured $K_{s}$ and Jaky's approximation; moreover a smooth relation between $K_{\mathrm{s}}$ and $\phi_{\mathrm{i}}$ or $\phi_{\mathrm{e}}$ is absent, which indicates that the same is true for $K_{0}$. This means that $K_{\mathrm{s}}$ cannot be predicted using only the internal angle of friction. Although it is likely that $\phi_{\mathrm{i}}$ has an influence on the value of $K_{\mathrm{s}}$, other parameters will play a role, such as the actual strain, the particle size and shape. A good approximation of $K_{\mathrm{s}}$ can only be found by measurements comparable to those described here. If this is not possible, a value of $K_{\mathrm{s}}=0.4$ is as valid as the value of $K_{\mathrm{s}}$ calculated with Jaky's formula.

The value of $K_{\mathrm{s}} \rho g z$ is an upper limit of the wall stress, since shear stresses along the wall reduce the normal wall stress. It would be interesting to investigate the limiting value of $K_{\mathrm{s}}$ at repeated loadings. As mentioned, $K_{\mathrm{s}}$ seems to diminish slightly when the sample 
is reloaded. With solids stored in mammoth silos, repeated loading is the rule rather than the exception.

With this method a number of tests can be performed. For instance, the influence of time on a loaded sample can be estimated in two ways, by measuring either creep or relaxation.

\section{Symbols}

d unstretched wire diameter

$D \quad$ unstretched membrane diameter

$s \quad$ unstretched pitch

$E_{\mathrm{r}} \quad$ radial wall stiffness

$E_{\mathrm{w}} \quad$ circumferential wall stiffness

$K_{0} \quad$ stress ratio of horizontal to vertical stress with no horizontal strain

$K_{\mathrm{s}} \quad$ stress ratio of horizontal to vertical stress with limited horizontal strain

$\Delta R \quad$ resistance change

$\Delta V \quad$ voltage change

$V \quad$ bridge voltage

$C, C^{\prime}, C^{\prime \prime}$ material constants

$\epsilon_{\mathrm{r}} \quad$ radial strain

$\epsilon_{\mathrm{w}} \quad$ circumferential strain

$\sigma_{\mathrm{r}} \quad$ radial stress

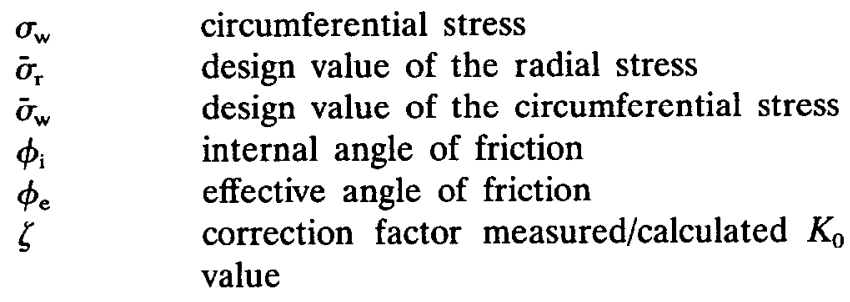

\section{References}

1 F.J.C. Rademacher, G. Haaker and N.P. Kruyt, Silo's en Feeders (in Dutch), University of Twente, Enschede, 1990.

2 T.W. Lambe and R.V. Whitman, Soil Mechanics, MIT Press, Cambridge, MA, 1979.

3 R.H. Kuerbis and Y.P. Vaid, Geotechnical Testing J., 13 (1990) 361.

4 J. Jaky, J. Soc. Hungarian Architects Eng., (1944) 355.

5 E.T. Miller, Stresses and Strains in an Array of Elastic Spheres, Report R 53-59, US Army Eng. Waterways Exp. Station, 1963.

6 M. Hetényi, Handbook of Experimental Stress Analyses, Wiley, New York, 1950.

7 P.L.L. van Leeuwenstijn, MSc. Thesis, University of Twente, Enschede, Dec. 1992.

8 DIN 1055 Part 6, German Standards Institute, Berlin, May 1987.

9 L.A. van Wijk, Ph.D. Thesis, University of Twente, Enschede, Nov. 1993, pp. 52-62. 